\title{
Comunicación estratégica aplicada en proyectos de implantación tecnológica en el Estado uruguayo
}

\author{
Strategic communication applied in technology implementation projects in \\ the Uruguayan State
}

\author{
Daniel Ottado \\ Universidad de la República \\ daniel.ottado@comunicacion.edu.uy
}

Recibido: 10 de septiembre 2013 Aceptado y Publicado: 24 de noviembre de 2013

\begin{abstract}
Resumen
En los últimos años los estados latinoamericanos han experimentado un cambio significativo en su gestión al haber incorporado tecnologías de gestión y de la información y la comunicación (TIC), con el objetivo de mejorar en eficiencia, confiabilidad y transparencia y de fortalecer el vínculo Estado ciudadano con mayor facilidad para realizar trámites y a la vez brindar a éste la información necesaria al momento de rendir cuentas de la administración realizada.

Este proceso de modernización de las administraciones públicas implica profundos cambios en materia de conocimiento sobre el manejo y uso de las tecnologías, en los procesos, el estilo de gestión, las estructuras y las culturas de las organizaciones, las cuales impactan directamente sobre las personas que trabajan en ellas, elemento esencial y determinante de cualquier organización. Por tal motivo, el factor humano se torna un imprescindible foco de atención para quienes gestionan el cambio, dentro y fuera de los organismos del Estado.

En este corto pero intenso período de cambios tecnológicos en el Estado uruguayo, la administración pública en general y los equipos que trabajan en la implantación de estas tecnologías, han experimentado un proceso de aprendizaje en materia de implantaciones, uso y apropiación tecnológica de estos nuevos sistemas.

El presente trabajo recorre parte de este proceso de implantaciones que fueron desde la aplicación más "dura" del conocimiento tecnológico donde la solución se reducía a la implantación y correcto uso de la herramienta informática, hasta la introducción de metodologías de gestión del cambio y particularmente de comunicación estratégica en cada uno de los proyectos de implantaciones tecnológicas con el propósito de lograr apropiación de las mismas y la sustentabilidad de estos proyectos.
\end{abstract}


Asimismo, este documento pretende generar un espacio de intercambio de experiencias, análisis y reflexión sobre las diferentes estrategias y herramientas de comunicación que pueden contribuir al éxito en la gestión del cambio dentro y fuera de nuestras organizaciones públicas, con foco en la atención al ciudadano.

\section{Summary}

In recent years, Latin American states have undergone a significant change in their management by having incorporated management and information and communication technologies (ICT), with the aim of improving efficiency, reliability and transparency and strengthening the citizen state link with greater ease to carry out procedures and at the same time provide him with the necessary information at the time of rendering accounts of the administration carried out.

This process of modernization of public administrations implies profound changes in knowledge about the management and use of technologies, in the processes, management style, structures and cultures of the organizations, which directly impact the people who They work in them, an essential and determining element of any organization. For this reason, the human factor becomes an essential focus of attention for those who manage change, inside and outside State agencies.

In this short but intense period of technological changes in the Uruguayan State, the public administration in general and the teams that work on the implementation of these technologies have undergone a learning process regarding the implementation, use and technological appropriation of these new systems .

The present work covers part of this implementation process that went from the "hardest" application of technological knowledge where the solution was reduced to the implementation and correct use of the computer tool, to the introduction of change management methodologies and particularly of Strategic communication in each of the technology implantation projects in order to achieve ownership of the same and the sustainability of these projects.

Likewise, this document aims to generate a space for the exchange of experiences, analysis and reflection on the different communication strategies and tools that can contribute to the success of change management within and outside our public organizations, with a focus on citizen service.

Palabras clave: Comunicación estratégica; Tecnología; Cambio; Uruguay.

Keywords: Strategic communication; Technology; Change; Uruguay. 


\section{Comunicación estratégica aplicada en proyectos de implantación tecnológica en el Estado uruguayo}

El Discurso que ofrecía el Dr. Tabaré Vázquez el 1ํ de marzo de 2005, en el acto realizado en la explanada del Palacio Legislativo, el día que asumió como Presidente de la República, incorporaba dos conceptos que serían buque insignia de su gestión: modernización y reforma del Estado ( $\mathrm{La}$ onda digital, disponible en URL: http://www.laondadigital.com/laonda/laonda/473/C4.htm [consulta 20 de abril de 2013]). Posteriormente, en la administración José Mujica, ambos conceptos se mantuvieron no ya con una presencia tan fuerte en el discurso institucional, pero sí a través de acciones concretas, como es el caso de proyectos de implantación tecnológica para la mejora de la gestión del Estado.

Estas acciones que se vienen desarrollando en Uruguay, se enmarcan en una contexto latinoamericano y mundial de la última década en el que los estados han incorporando tecnologías de la información y la comunicación (TIC) en la gestión pública con el fin de mejorar la eficiencia, la confiabilidad, la transparencia y el fortalecimiento del vínculo Estado y ciudadano, a través de mejores servicios de trámites e información que éste brinda a la ciudadanía.

Este proceso de modernización de las administraciones públicas a través del uso de las TIC está impactando directamente sobre la normativa, los procesos, el estilo de gestión, las estructuras y las culturas de las organizaciones. Por ende, impactan en las personas que trabajan en ellas, elemento esencial y determinante de cualquier organización.

Si bien las personas resultan ser un factor imprescindible en cualquier proceso de cambio dentro de una organización, no siempre se ha puesto la debida atención a este elemento al momento de realizar una implantación tecnológica.

Los últimos años han sido muy intensos en materia de innovaciones tecnológicas y actualmente existe una amplia diversidad de soluciones informáticas para la gestión de la administración pública, muchas de los cuales se han implantado en diversos organismos del Estado.

Uno de esas soluciones es el sistema de expediente electrónico que comenzó a implantarse en el Estado uruguayo a fines de 2009. Estas experiencias llevaron a los equipos que trabajaron estos proyectos a un proceso de aprendizaje producto del ensayo y error en el terreno. 
Dado el importante número de experiencias realizadas en esta materia dentro del Estado, y al no poder sintetizar en un trabajo de esta naturaleza todo el camino recorrido, se tomó $u$ caso que recoge parte del proceso realizado, la evolución hacia un salto cuantitativo y cualitativo en materia de éxito en las implantaciones tecnológicas, y, hasta cierto punto, un cambio paradigmático en la forma de entender lo que implica una implantación tecnológica.

\section{Un marco de referencia}

Para incursionar en este terreno, nos basamos en algunos conceptos sobre comunicación estratégica y gestión del cambio que nos permitirán tener una referencia conceptual para el análisis de estas experiencias mencionadas anteriormente.

A diferencia del orden en el que está redactado el título de esta ponencia, tomaremos algunas definiciones de lo que, según algunos autores, se entiende como gestión del cambio.

\subsection{Qué entendemos por gestión del cambio}

Como señala Lladó, Eduardo y Ronco, Emilio, cuando hablamos de gestión del cambio nos referimos a aquellos cambios que no son espontáneos, sino que son planificados (Lladó y Ronco, 2001). En los cuales interviene un agente de cambio que tiene la función de conducir este proceso en una dirección previamente definida y determinada.

Asimismo, tomamos de estos autores el concepto de cambio impuestos y cambio participativo que nos será útil más adelante, para dilucidar algunas acciones concretas en materia de implantaciones tecnológicas. Sobre estos conceptos, propiamente, el cambio impuesto es aquel que viene dirigido de manera vertical descendente y que no deja lugar a que las personas involucradas en este proceso puedan realizar algún tipo de modificación. Por el contrario, en el modelo participativo, las personas involucradas "intervienen en el proceso de decisión y de implantación del mismo" (Lladó y Ronco, 2001, p. 30)

Para los autores anteriormente mencionados la decisión de qué modelo de intervención tomar no es fortuita, sino que de ella depende el éxito de la gestión del cambio. Y ellos lo sintetizaron en la siguiente fórmula: el cambio depende de la calidad del equipo técnico y que sea aceptado por parte de los involucrados.

Al momento de pensar en el diseño de un proceso de gestión del cambio, Kurt Lewin, uno de los primeros investigadores en esta materia, identifica tres fases de este proceso que denomina: descongelamiento, cambio y congelamiento. El primero refiere a concientizar 
sobre la necesidad del cambio mediante la generación de un clima que permita cuestionar la situación actual. El segundo es incidir en las personas para que cambien sus conductas y en las diferentes "subsistemas" de la organización que permitan apoyar y reforzar el proceso de cambio. Finalmente, el congelamiento se da cuando los individuos han interiorizado el proceso de cambio, generando los hábitos y conductas previstas. (Ibídem).

Kurt Lewin plantea un modelo de análisis de campo de fuerzas, en el cual se pueden identificar fuerzas facilitadoras y limitadoras del cambio. Este mapa permitirá al agente de cambio actuar sobre las fuerzas limitadoras que resisten el cambio y actuar en consecuencia (Ibídem).

Finalmente, otro autor que nos brinda un marco conceptual sobre cambio organizacional, es John Kotter, que a lo largo 20 años de experiencia en la materia, expone algunos elementos que surgen de la empírica.

John Kotter señala que, para que se produzcan cambios en las organizaciones depende de dos componentes: el primero consiste en un proceso con múltiples etapas y el segundo refiere al liderazgo como, a fuerza que impulsa el cambio.

El proceso que el autor propone contiene ocho pasos: 1. Infundir el sentido de premura, 2. crear la coalición conductora, 3. desarrollo de una visión y una estrategia, 4. comunicar la visión de cambio, 5. facultar a los empleados para emprender acciones de largo alcance, 6. generar logros a corto plazo, 7. consolidar las ganancias y generar cambios y 8 . arraigar los enfoques en la cultura (Kotter, 1997). Si bien este planteo proviene del mundo empresarial, consideramos que varios de estos elementos son utilizados también en los procesos de cambio dentro de las organizaciones públicas que no pertenecen al sector empresarial, como lo comprobaremos más adelante.

De forma muy sintética trataremos de desarrollar los elementos más significativos de cada uno de estos ocho pasos a los efectos de dar un pantallazo conceptual sobre a qué refiere cada uno de ellos. Estos resultarán de importancia al momento de contrastarlos con los casos concretos de implantación tecnológica que hemos citado al inicio de este trabajo (Ibídem).

1. El sentido de premura que señala Kotter refiere a mostrarle a las personas que forman parte de la organización la urgencia del cambio. Por ejemplo, mediante la identificación de potenciales amenazas u oportunidades que podrían generarse en el futuro, tener la visión de actores externos a la organización como clientes o usuarios. 
2. Crear la coalición conductora consiste en integrar un equipo con capacidad de liderazgo, sólido técnicamente, con un alto grado de confianza y comprometido con los objetivos establecidos en el proyecto. Kotter lo ve como un elemento clave al inicio de cualquier proyecto.

3. El desarrollo de una visión y una estratégica tiene que ver con "una imagen de futuro", el rumbo que se debe trazar y el motivo de por qué la gente debe de esforzarse. Una visión cargada de valores y de fácil y rápida descripción.

4. Comunicar la visión del cambio consiste en lograr que la gran mayoría de las personas que forman parte de la organización comprendan de igual forma "sus objetivos y dirección". Esto implica tener la capacidad de escuchar y ser escuchado, hablar a menudo de la visión del cambio cuando la situación lo permita y atarla inclusive a los aspectos operativos. Al mismo tiempo ser coherente entre el discurso y la acción, lo que comúnmente se conoce como "predicar con el ejemplo".

5. Facultar a los empleados para emprender acciones a largo alcance se trata de eliminar la mayor parte de las barreras que pueden dificultar el accionar de los para el cambio. Obstáculos que se pueden dar en la estructura orgánica y su jerarquía o en las habilidades interviniendo en la capacitación de las personas para superar ese escollo.

6. Generar logros a corto plazo señala el autor sirven para apalancar el proceso de cambio, generar confianza, redoblar la motivación de quienes están comprometidos y demostrar a quienes se resisten al cambio que este proceso de cambio avanza y es irreversible. Dado que las grandes transformaciones llevan tiempo alcanzar objetivos al corto plazo que contribuyan a la meta final, resultan ser un aliciente indispensable para creer la viabilidad y factibilidad del proyecto.

7. Consolidar las ganancias y generar más cambios resulta un punto clave en el que se reconocen los hitos alcanzados, pero no se pierde de vista el objetivo principal de largo alcance. Es presentar nuevos desafíos y recordar a los involucrados que aún hay camino que recorrer para alcanzar el cambio profundo. Para ello hay que establecer nuevos desafíos a la organización.

8. Arraigar la nueva cultura es el último de este proceso de cambio que tiene por finalidad la "sostenibilidad del cambio" y la instauración de una cultura del cambio. Esto es posible no solo a través de recordar permanentemente mostrar los valores de la visión, sino también, procurar que los líderes sigan apoyando el cambio y 
trabajando en el recambio de los mismos para que no se pierda la cultura de cambio instaurada.

Con el marco conceptual presentado en materia de gestión del cambio estaríamos en condiciones de avanzar hacia la conceptualización del término comunicación estratégica del que trata la primera parte del título de esta ponencia.

\subsection{De qué hablamos cuando hablamos de estrategia}

Para abordar el concepto de comunicación estratégica tomamos la definición que plantea Rafael Alberto Pérez González. Este autor señala que la comunicación es interacción simbólica y que la misma puede ser calificada como estratégica cuando ocurren al menos dos características: por un lado que "se produce en una situación de juego en la que intervienen varios jugadores", al menos dos, y por otro, "que tienen que tomar decisiones antes la incertidumbre que generan otros jugadores” (Pérez González, 2006, p.455).

En este sentido, tener en cuenta el juego de otros jugadores en la partida es determinante ya que éstos pueden incidir en lo que ambicionamos. Por ende, según este autor, la estrategia se transforma "esencialmente en un acto de coordinación" (Pérez González, 2006, p.456).

Este enfoque contempla que las personas tienen la libertad de elegir entre un conjunto de posibilidades para construir su destino y que al mismo tiempo la libertad de elegir no necesariamente se transforma en libertad para actuar, ya que la misma depende de los recursos de diversa índole con los que contemos.

Por lo anteriormente dicho, en este marco la comunicación no se produce de forma espontánea, sino que hay una clara intencionalidad en donde se evalúan los contextos en donde esta se produce y en función de un objetivo dado. A partir de allí se configura el discurso. Persuadir e influir son parte de esta acción comunicativa. La estrategia se selecciona en función de las intenciones del comunicador.

Para estos fines, Rafael Alberto Pérez propone una serie de mensajes que denomina "ideas fuerza" que configuren más de una estrategia a elegir y una mezcla de sistemas comunicativos que pueden articularse, como podrían ser mailing, redes sociales, entre otros (Ibídem).

La comunicación estratégica implica planificar, ejecutar, controlar, evaluar y realizar acciones correctivas del plan y la estrategia desarrollada. Por lo tanto según este autor: "la comunicación estratégica debe ser entendida como una sucesión de jugadas en el espacio tiempo y no como una única partida" (Ibídem). 
Finalmente, desde este maro conceptual, la comunicación estratégica sirve, en palabras de Rafael Alberto Pérez, "para encauzar el poder de la comunicación y así incidir en los resultados de la partida que estamos jugando en la orientación que marcan nuestros objetivos". (Ibídem)

\subsection{Cambiando la forma de entender las implantaciones tecnológicas}

El caso que citaremos en este trabajo, responde a una de las experiencias de implantación tecnológica de Expediente Electrónico apoyadas por la Agencia para el Desarrollo del Gobierno de Gestión Electrónica y la Sociedad de la Información y del Conocimiento (AGESIC) en un organismo público del Estado uruguayo.

El caso presenta los elementos más significativos a nuestro entender y que dan cuenta de las diferencias de enfoque de la implantación en materia de comunicación y gestión del cambio.

AGESIC se creó en 2008. Es una unidad ejecutora que depende de la Presidencia de la República y que tienen como objetivo "procurar la mejora de los servicios al ciudadano, utilizando las posibilidades que brindan las Tecnologías de la Información y las Comunicaciones (TIC)." (AGESIC en URL: http://www.agesic.gub.uy/innovaportal/v/19/1/agesic/que_es_agesic.html [consulta 2 de febrero de 2013]).

\section{Las primeras experiencias de implantación de Expediente Electrónico}

En 2008 comenzaron las primeras experiencias de implantación de Expediente Electrónico (EE en diferentes organismos del Estado. A partir de allí, la implantación de EE se incrementó cuantitativa y cualitativamente la implantación de este sistema en otros organismos del Estado. 


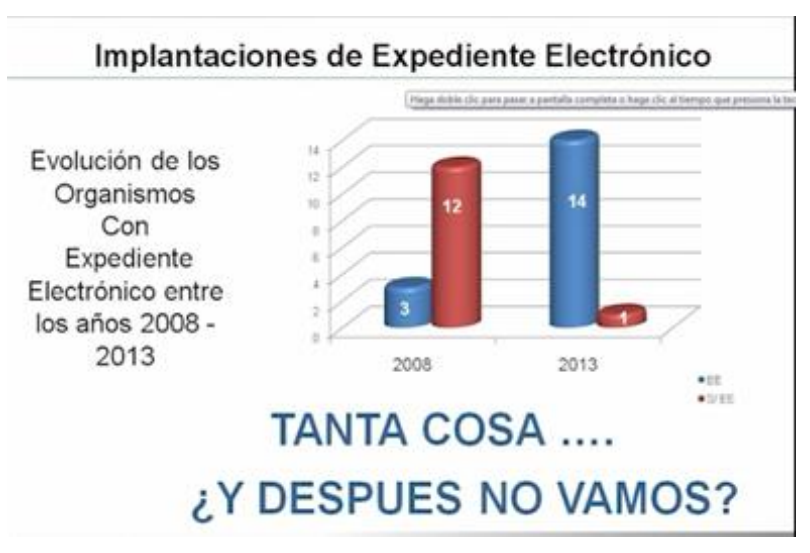

Fuente: AGESIC

AGESIC tue creada en diciembre de 2005 con la denominacion "Agencia para el Desarrollo de Gobierno Electrónico" (Artículo N 72 - Ley 17.930) y su funcionamiento fue reglamentado en junio de 2006 (Decreto 205/006) (AGESIC en URL: http://www.agesic.gub.uy/innovaportal/v/19/1/agesic/que_es_agesic.html [consulta 2 de febrero de 2013]).

Para la implantación de tecnología, la Agencia trabaja en coordinación con los organismos públicos y usualmente con una empresa que mediante llamado público es seleccionada como la consultora que participa de este proceso.

Las primeras implantaciones no incluyeron dentro de su requerimiento gestión del cambio y comunicación en estos procesos. Esto se puede apreciar en la invitación a presentaciones de interés que fueron publicadas por entonces y en donde estos dos componentes no están explicitados.

En las publicaciones de pliegos posteriores aparecerán los ítems comunicación y gestión del cambio dentro de los mismos para la implantación de EE:

\section{Implantación de EE en Ministerio del Interior: un caso de éxito}

El transcurso del tiempo, la dedicación a evaluar, la apertura a discutir los procesos y los resultados, las perspectivas multidisciplinarias sobre un mismo fenómeno, conducen a la reflexión y a la generación de oportunidades para mejorar los procesos, en este caso, de implantaciones tecnológicas. 
Así fue en parte este derrotero que seguramente ha transitado el Estado uruguayo. El hecho de incluir nuevos componentes como la gestión del cambio y la comunicación estratégica, contribuyeron a mejorar las posteriores implantaciones.

Un caso de éxito en la materia, es la experiencia transitada por el Ministerio del Interior (MI). Esto puedo apreciarse en el V Encuentro Nacional de Gobierno Electrónico que se realizó en los días 5 y 6 de diciembre de 2012 en Montevideo. En esta oportunidad representantes de la mencionada agencia y representantes del MI expusieron la experiencia de implantación de EE en el referido organismo. El punto de partida de partida de esta aseveración radica ya en que el pliego del llamado incluye como dos de sus componentes la gestión del cambio y la comunicación.

En la presentación denominada Expediente Electrónico: Evolución de las implantaciones en los Organismos del Estado se pueden identificar las buenas prácticas y las lecciones aprendidas en esta implantación, en donde realizan un fuerte énfasis en los procesos de gestión del cambio como un componente esencial para una implantación tecnológica (AGESIC en

URL: http://www.agesic.gub.uy/innovaportal/v/2423/1/agesic/presentaciones.html [consulta 2 de febrero de 2013]).

Dentro de los los desafios en materia de comunicación y gestión del cambio que el Ministerio le presentaba al proyecto, se encontraba:

- la jerarquía orgánica: es una organización fuertemente jerárquica y donde las dinámicas de trabajo que se proponen en un proceso de esta naturaleza requiere que se trabaje de forma coordinada entre personal de diferentes escalafones jerárquicos, que son quienes integran el equipo de proyecto del organismo.

- La dispersión de unidades en todo el país y el número de funcionarios que las integran, genera, entre otras cosas, dificultades de cercanía al momento de sensibilizar, concientizar y responder a las dudas que se generan sobre el proyecto.

- el descreimiento de las personas en los procesos de cambio y en la ejecuciòn exitosa del proyecto.

Dada la maginitud del proyecto se ajustó el plan inicial con un plan piloto para poder ser abarcado y lograr pequeños hitos que fuesen solidificando el proyecto a largo plazo. En tal sentido, se establecieron etapas que comprendian la implantación en diferentes unidades para cada una de ellas. 
Concomitantemente, a medida que se sucedian las etapas, se iba adelantando trabajo en otras unidades mediante la capacitaciòn en alfabetización digital y el uso de equipoamiento como escáners, computadoras, entro otros.

Este proceso generó y el alcance de logros intermedios, generó paulatinamente, la aceptación del personal y la confianza en el grupo de pares que formó parte del equipo que liderò el cambio. Hubo también un cambio de actitud hacia la herramienta informática, ya que muchos funcionarios comenzaron a contactarse con el equipo de cambio para saber cuándo serian las capacitaciones en su sector y algunas unidades se postularon por iniciativa propia a formar parte del proceso, señalan las expositoras.

En materia de comunicación estratégica se definió una idea fuerza que ha acompañado desde el inicio la implantación de EE en el Estado. La misma consiste en: "Estás listo para algo más simple". Como bajada de eslogan, un texto señala el proceso en el que se encuentra Uruguay en materia de información, trámites y servicios por internet. Así también los avances sobre gobierno electrónico que se han realizado, las facilidades que brinda la tecnología y la dirección a la que apunta este proyecto al largo plazo. (AGESIC en URL: http://www.agesic.gub.uy/agesic/boletines/newsletter/boletin web item.jsp?newsletter=1058 \&contentid $=1024 \&$ modo $=$ newsletter $\&$ site $=1 \&$ newsletter $=\&$ folder $=933 \&$ year option $=2010 \&$ month $=16$ modor $=$ newsletter\&addressbook $=1035$ [consulta 8 de febrero de 2013]).

Al mismo tiempo, estas acciones comunicación han sido acompañadas por otras en diferentes canales: afiches, mailing, pop up con un contabilizador de tiempo de lanzamiento 0 recordaciòn de eventos que se abren apenas las personas encienden sus computadoras en el trabajo, intervenciones en las oficinas con manualidades y figuras que recuerdan la ruta recorrida y hacia dónde se dirigen.

Como lecciones aprendidas para una implantación de esta naturaleza, el MI destaca la importancia de contar con :

- un patrocinador de jerarquía, respaldo y recursos necesarios

- un equipo de proyecto fuerte, motivado, comprometido y con solidez técnica.

- el apoyo de las autoridades de las unidades ejecutoras en este proceso y la facilitación de la información para que llegue a todos los involucrados.

Por su parte, AGESIC destaca en este proceso de aprendizaje las siguintes lecciones aprendidas: 
- El organismo debe estar preparado para recibir la herramienta. En este sentido debe demostrar interés en su implantación y demostrar que cuenta con un equipo con capacidades tècnicas y voluntad de transitar ese cambio.

- El proyecto debe ser del organismo. Esto refiere a que para avanzar en el cambio, éste debe sentirlo como propio.

- El foco debe estar en la gestión del cambio y no en la herramienta.

- El organismo debe conocer la herramienta antes de su implantación: debe de haber una capacitación inicial al grupo de trabajo antes de comenzar a personalizar la heramienta. Esto genera un lenguaje común entre las partes involucradas.

- Realizar implantaciones con ruta libre - dejar capacidades para continuar avanzando: vamos con relevamiento de rutas que si no son el $100 \%$ de la ruta real genera confusión en el organismos, si es el funcionario o la herramienta. Después se decide si el organismo toma la ruta sugerida.

A continuación transcribimos parte del discurso que realizó la Ing. Sabrina Trotta, representante por MI, en ese encuentro nacional de Gobierno Electrónico en diciembe de 2012, que refleja el aprendizaje realizado en esta experiencia: "Lo decisivo del proyecto no es saber configurar la herramienta, saber cómo funciona, capacitar a la gente, sino hacer entender a la gente que este proyecto les va a simplificar la vida en su trabajo diario. Una vez que los convensamos de eso, esa persona va a ser un vocero, va a ser un gestor del cambio, un factor de cambio y va a ser el que corra la voz entre sus compañeros de lo bueno que está esto. Esa es la única manera que el proyecto salga. Cuando me lo dice el compañero de al lado, ahí sí, le creo."

Y finalmente agrega: "Pero no hay que mentir, decirle que al principio va a costar, decirle sobre la curva de aprendizaje de la herramienta, que va a utilizar tecnología que nunca utilizó en su vida y a manejar más de un sistema a la vez. Pero una vez sorteadas todas esa cosas el trabajo se disminuye y se ordena muchísimo".

Al cierre de su alocución, expresa la siguiente frase que resume la forma en que esta organización entiende que debe funcionar este proyecto: "La consultora apoya, AGESIC respalda, Ministerio del Interior trabaja". (audio registrado en el evento del $\vee$ Encuentro Nacional de Gobierno Electrónico, 6 de diciembre de 2012)

En este discurso podemos apreciar algunos aspectos en donde la comunicación estratégica tiene un rol fundamental en estos procesos de gestión del cambio en implantaciones tecnológicas. Por ejemplo cuando refiere a"hacer entender al otro" nos recuerda a ese enfoque hermenéutico y cultural de la comunicación del que nos habla Rafael Alberto Pérez, 
donde las personas mediante la interpretación y la extracción de significación, buscan reducir la incertidumbre (Pérez González, 2006, p.456). Es a una de las cosas que debemos contribuir desde esta perspectiva de comunicación estratégica.

Al mismo tiempo, la presentadora, nos habla de ese componente relacional de la comunicación en donde la acpetación de un cambio, como es este el caso, proviene o tiene mucha incidencia de quien venga el mensaje y como es interpetado y en donde aún en tanta tecnología el boca a boca o boca oído juega un rol muy importante en nuestras organizaciones "modernas".

Y por último la franqueza o al menos brindar el mayor grado de veracidad en la información que se solicita. El dicho popular "la mentira tiene patas cortas" toma un vital significado para construir relaciones de confianza, afectivas y duraderas. Es crucial para el individuo saber lo que tiene por delante. Eta forma de comunicación, contribuye a generar cimientos vinculares que serán soporte para emprender nuevos desafíos dentro de la organización.

\section{A modo de conclusión}

Como hemos visto a lo largo de este trabajo, muchas de los elementos conceptuales expuestos por diferentes autores en materia de gestión del cambio y comunicación estratégica aplicada a la gestión del cambio, se han visto reflejados en las experiencias citadas sobre implantación de EE en los organismos públicos del Estado. El contemplar su uso ha sido en gran medida un factor determinante para alcanzar el éxito de una implantación tecnológica.

La planificación en etapas, la consecución de pasos de un proceso de gestión del cambio, el liderazgo, y la comunicación entendida como estratégica, nos muestran empíricamente que esta metodología de trabajo resulta ser sensiblemente más adecuada para alcanzar el éxito. Al menos en sociedades democráticas donde las personas tienen la posibilidad de elegir y cierto margen de acción para poder actuar en consecuencia.

Seguramente, hay otras formas de gestionar los cambios, de forma menos participativa y más direccional, impuesta y es posible. Pero si queremos que la gente se sienta parte, se comprometa, aporte ideas y se apropie de un proyecto es necesario desarrollar un proceso de cambio que se adecue a esta necesidades y una comunicación estratégica que contribuya a tales fines. 


\section{Bibliografía}

Capriotti, Paul (1999) Planificación Estratégica de la Imagen Corporativa. Barcelona: Ariel.

Capriotti, Paul (2007) Gestión de la Marca Corporativa. Buenos Aires: La Crujía.

Capriotti, Paul (2009) Branding Corporativo: Fundamentos para la gestión estratégica de la Identidad Corporativa. Santiago de Chile: Colección de Libros de la Empresa. Disponible en URL: http://www.analisisdemedios.com/branding/BrandingCorporativo.pdf [consulta 3 de enero de 2013].

Chaves, Norberto (2006) La Imagen Corporativa. Barcelona: Gustavo Gili.

Kotter, John P. (1997) EL líder del cambio., México McGraw-Hill

Lladó, Eduardo y RONCO, Emilio (2001) Aprender a gestionar el cambio. Barcelona: Bosch.

Pérez González, Rafael Alberto (2006) Estrategias de comunicación. España: Ariel Comunicación.

AGESIC en URL: http://www.agesic.gub.uy/innovaportal/v/19/1/agesic/que_es_agesic.html [consulta 2 de febrero de 2013]).

AGESIC en URL: http://www.agesic.gub.uy/innovaportal/v/2423/1/agesic/presentaciones.html [consulta 2 de febrero de 2013].

AGESIC en URL:

http://www.agesic.gub.uy/agesic/boletines/newsletter/boletin_web_item.jsp?newsletter=1058 \&contentid $=1024 \&$ modo $=$ newsletter $\&$ site $=1 \&$ newsletter $=\&$ folder $=933 \&$ yearoption=2010\&month=16modor=newsletter \&addressbook=1035 [consulta 8 de febrero de 2013].

La onda digital, disponible en URL: ttp://www.laondadigital.com/laonda/laonda/473/C4.htm [consulta 20 de abril de 2013]).

\section{Forma de citar este artículo en bibliografías}

OTTADO, D. (2013) "Comunicación estratégica aplicada en proyectos de implantación tecnológica en el Estado uruguayo" se encuentra publicado en Revista PANGEA (ISSN: 2172 - 3168); Año 2013, $N^{\circ} 2$, en páginas 89 a 102. Red Académica Iberoamericana de Comunicación. Recuperado el _ de _ de 2_ de: http://www.revistapangea.org 